Revue de l'Institut des langues et cultures

d'Europe, Amérique, Afrique, Asie et Australie

$38 \mid 2020$

Chant et nation : de la culture populaire à la culture savante

\title{
Traduire les chansons de Bob Dylan : enjeux de transfert culturel
}

Translating the Songs of Bob Dylan: The Question of Cultural Transfer

Jean-Charles Meunier

\section{OpenEdition}

Journals

Édition électronique

URL : http://journals.openedition.org/ilcea/9862

DOI : $10.4000 /$ ilcea.9862

ISSN : 2101-0609

Éditeur

UGA Éditions/Université Grenoble Alpes

Édition imprimée

ISBN : 978-2-37747-172-0

ISSN : 1639-6073

Référence électronique

Jean-Charles Meunier, "Traduire les chansons de Bob Dylan : enjeux de transfert culturel », ILCEA [En

ligne], 38 | 2020, mis en ligne le 31 janvier 2020, consulté le 31 janvier 2020. URL : http://

journals.openedition.org/ilcea/9862 ; DOI : 10.4000/ilcea.9862

Ce document a été généré automatiquement le 31 janvier 2020.

(c) ILCEA 


\section{Traduire les chansons de Bob Dylan : enjeux de transfert culturel}

Translating the Songs of Bob Dylan: The Question of Cultural Transfer

Jean-Charles Meunier

\section{Introduction}

1 La chanson « Motorpsycho Nitemare ", de Bob Dylan, est sortie sur l'album Another Side of Bob Dylan (1964a). Musicalement, elle a subi l'influence du talking blues (cf. Margotin \& Guesdon, 2015 : 124), une forme que Dylan a plusieurs fois utilisée $(1963,2010)$ et qui peut être définie comme un style de musique blues dans lequel les paroles sont plus ou moins parlées plutôt que chantées (cf. Stevenson, 2010). Une partie significative du sens de « Motorpsycho Nitemare » repose sur une bonne connaissance du contexte culturel états-uniens des années 1960. Il est fait référence à l'anticommunisme de la guerre froide, au cinéma américain, et à une forme de comique qui s'inscrit à la fois dans l'espace et dans le temps. Traduire cette chanson pour qu'elle soit chantée en français comporte par conséquent des difficultés qui vont au-delà des obstacles habituels liés à la traduction de chansons, à savoir la nécessité de préserver le rythme et, si possible, les rimes. Dans cet article, nous nous intéresserons à deux traductions françaises : celle d'Hugues Aufray, enregistrée sur l'album Aufray chante Dylan (1965) et celle de l'artiste suisse Sarclo, enregistrée en trio avec Albert Chinet et François Pierron (2017). Pour transférer dans la traduction française ce qui fait l'essence de cette chanson, quatre éléments caractéristiques en particulier sont à prendre en compte: l'humour burlesque, les références au film Psycho [Psychose], sorti en salles quatre ans avant la chanson (Hitchcock, 1960), la satire du maccarthysme et la plaisanterie sur les commis voyageurs qui forme la structure de la chanson. 


\section{Le slapstick : chanter le comique visuel}

2 L'une des formes de comique employées par Dylan dans cette chanson est le slapstick, mot qui vient du nom d'un bâton servant à imiter le bruit d'un coup, appelé « bataccio », dans la commedia dell'arte. Ce type de comique est défini de la façon suivante dans le Dictionary of media and communications : " comedy revolving around crude practical jokes " [" comédie qui tourne autour de farces et de comique grossier »] (Danesi, 2009: 272). Cette forme d'humour se trouve en France dans le cinéma de Jacques Tati ou Louis de Funès, par exemple, et aux États-Unis dans les films de Charlie Chaplin ou Laurel et Hardy. Ce comique visuel est présent dans la chanson en particulier à travers la confrontation entre le narrateur et le vieux fermier, qui est décrite comme une bagarre de saloon: "swung at me with all his might » [«il a essayé de toutes ses forces de me donner un coup de poing " (ma traduction $\left.{ }^{1}\right)$ ], "Landed fully blast in his garden flowers » [«j'ai atterri à toute vitesse dans les fleurs de son jardin »], « And stuck a gun into my guts » [« et il a m’a collé son fusil dans le ventre »].

3 Aufray et Delanoë ont traduit «swung / At me with all his might » de la façon suivante : "il me tomba sur le dos à bras raccourcis ». Plutôt que de traduire précisément le sens de "with all his might», les traducteurs ont fait le choix d'utiliser l'expression imagée française "à bras raccourcis", ce qui, d'une part, convoque l'image potentiellement drôle qui se cache derrière la métaphore et, d'autre part, peut faire référence au chef Abraracourcix, dans la série de bandes dessinées Astérix le Gaulois, dont le premier numéro a été publié quelques années avant cette traduction, et dans laquelle il est également beaucoup question de bagarres (Goscinny \& Uderzo, 1959). La seconde expression, « landed fully blast / In his garden flowers », a été traduite ainsi : «j'atterris en tempête / Dans un pot de fleurs ». L'humour de l'image originale est conservé, mais la présence du " pot » dans le texte cible peut paraître surprenante, car on imagine mal le narrateur pouvoir tomber dans un pot. De plus, le texte original étant "garden flowers ", on s'attend plutôt à un parterre de fleurs qu'à des pots. Sarclo n'a changé aucune de ces deux traductions. En revanche, il a modifié la troisième. Là où Dylan chante «stuck $a$ gun into my guts ", Aufray chante "vient me braquer son canon / À la hauteur du menton ». L'image est différente, car ce n'est pas le menton qui est visé dans la chanson de Dylan, mais le choix de traduire "gun" par «canon" pour le faire rimer avec «menton» a pu être motivé par l'allitération en /k/ («braquer», "canon»), qui reproduit les allitérations et assonances du vers d'origine ("stuck», "gun», "guts»). Sarclo a choisi de garder une image plus proche de la version originale visuellement, traduisant «Vient me pousser son fusil / au milieu des abattis ». Le mot « abattis » fait référence aux pattes et ailerons, mais aussi au foie et au gésier de volaille. En outre, le mot évoque en même temps, par proximité phonétique, le mot "abats», un hyperonyme du mot « tripes ", qui est une traduction exacte du terme " guts ».

L'expression «charging down the stairs» [« il a descendu les escaliers quatre à quatre »] peut suggérer l'image du vieux « Teddy Roosevelt » Brewster qui, lui, monte chaque fois l'escalier en criant «chaaaaaarge » [ chaaaaaargez »] dans la pièce Arsenic and Old Lace, écrite par Joseph Kesselring en 1939 et portée à l'écran par Frank Capra (1944). Cette expression est traduite par «Le fermier soudain surgit», ce qui ne permet pas de conserver la connotation militaire du mot anglais «charge»[ [ il a descendu les escaliers en chargeant »]. En revanche, on pourrait considérer que ceci est compensé par l'utilisation du mot "soudain», qui rend son arrivée plus abrupte, ce qui est 
renforcé par l'allitération en /s/ (« soudain », «surgit»). Cette évocation visuelle du film de Capra illustre l'un des aspects caractéristiques de l'écriture de Bob Dylan, à savoir sa capacité à emprunter à la fois des éléments de culture littéraire et des références à la culture populaire. Christopher Rollason écrit, à juste titre :

Dylan's work is a particularly interesting case of a hybrid cultural object, the result of a fusion, not so much between a bipolarised "high" and "low" culture as between three different cultures-intellectual culture, mass culture, and folk or traditional culture. [L'œuvre de Dylan est un cas particulièrement intéressant d'objet culturel hybride, le résultat d'une fusion, non pas tant entre une culture réduite à deux pôles " culture intellectuelle » et « culture de bas étage », mais plutôt entre trois cultures différentes - la culture intellectuelle, la culture de masse, et la culture folklorique ou culture traditionnelle.] $(2007: 112)$

Il explique ensuite qu'il inclut la culture de masse et la culture traditionnelle dans un terme englobant qu'il appelle « popular culture » [« culture populaire»] (2007:113). Ces catégories ne sont cependant pas hermétiques et mutuellement exclusives. Dans le cas de "Motorpsycho Nitemare», on peut s'interroger sur la catégorie dans laquelle il faudrait ranger les références cinématographiques à Alfred Hitchcock et Federico Fellini, entre culture de masse et culture intellectuelle.

\section{De La Dolce Vita à Psychose : une chanson hantée par le cinéma}

Le choix d'orthographier incorrectement le mot « Nitemare » au lieu de "Nightmare » [ " cauchemar »] dans le titre de la chanson participe probablement de cette même dynamique de référence à la culture populaire. Le néologisme "Motorpsycho ", quant à lui, forme une paire minimale avec le mot «motorcycle » [« moto»], c'est-à-dire qu'ils

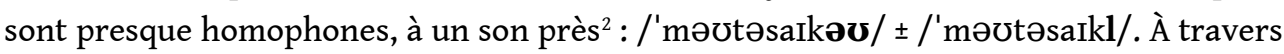
cette évocation de la moto, on peut voir le narrateur de la chanson comme un alter ego de Dylan, qui conduisait à l'époque une Triumph, jusqu'à son accident en 1966 (cf. Shelton, 2011 : chap. 11). La transformation de la seconde partie du mot, en revanche, est une référence au film d'horreur psychologique Psychose (Hitchcock, 1960), ce qui est rendu explicite par de multiples références, notamment la comparaison, dans le cinquième couplet, de Rita, la fille du fermier, avec « Tony Perkins », l'acteur qui joue le rôle de Norman Bates. Le mot-valise "Motorpsycho » permet donc à l'auteur, de façon très condensée, de nous indiquer qu'il s'agit d'un cauchemar sur la route, à l'image des nombreux films américains qui ont fleuri à la suite du chef-d'œuvre de Hitchcock, tels Duel, le premier long-métrage de Steven Spielberg (1971), et The Shining [Shining] (Kubrick, 1980), qui, comme Psychose, se déroule dans un « motel ", mot valise formé à partir du même mot «motor » (« motel » = « motor » + « hotel »). La traduction française du titre, "Cauchemar Psychomoteur", ne permet pas de transférer l'orthographe inhabituelle du mot "Nitemare». Il eût pour cela fallu traduire "Cochemar", par exemple, ou éventuellement «Cauchemare » pour faire référence au dernier mot du texte, «swamp » [ "marais ", " marécage »]. Le jeu sur le mot-valise « Motorpsycho", en revanche, est conservé, puisque le mot "Psychomoteur» constitue une paire minimale presque parfaite avec «cyclomoteur» en français: /psikJmotœR/ \pm / siklomotœR/.

6 L'évocation du nom de Tony Perkins est la référence la plus explicite au film de Hitchcock: «There stood Rita / Lookin' just like Tony Perkins » [ "Rita était là / On aurait 
dit Tony Perkins» ou « elle ressemblait comme deux gouttes d'eau à Tony Perkins »]. Aufray et Delanoë ont traduit « Elle me faisait de l'œil / Comme Tony Perkins ». Il s'agit probablement d'une mauvaise interprétation du verbe «looking», qui ne signifie pas ici « regarder » mais « ressembler à ». Sarclo a apporté une correction : « Elle souriait dans le noir / Comme Tony Perkins", ce qui correspond davantage au personnage de Norman Bates. En effet, il n'y a pas de scène dans Psychose où Norman Bates fait de l'œil à Marion Crane, la victime du célèbre meurtre sous la douche, interprétée par Janet Leigh. En revanche, le sourire terrifiant de Tony Perkins à la fin du film reste gravé dans la mémoire du spectateur, car il s'agit d'une des dernières images. L'auditeur peut donc imaginer l'effet de ce sourire dans le noir et s'identifier avec le narrateur. Ces deux traductions, cependant, impliquent une attitude particulière de la part de Rita, alors que le texte source, "Lookin' just like», ne fait qu'évoquer une ressemblance physique entre les deux personnages dans les yeux du narrateur. Lorsque le narrateur l'aperçoit pour la première fois dans le troisième couplet, Dylan chante «She looked like she stepped out of / La Dolce Vita ", ce qui est traduit en français par "Elle semblait tout droit sortie de / La Dolce Vita ", en référence au film de Federico Fellini (1960). Le contraste frappant entre ces deux comparaisons, toutes deux émanant de l'esprit du narrateur, semble donc refléter uniquement des hallucinations dues à l'évolution de son état psychologique au fil de la chanson.

Dans le couplet 5, Rita propose au narrateur de prendre une douche, comme Janet Leigh dans Psychose: "Would you like to take a shower? / I'll show you up to the door", ce qu'Aufray et Delanoë ont traduit par «viens prendre une douche / Je vais te montrer où c'est ». Sarclo a gardé la même traduction. En revanche, son interprétation de la chanson étant plus proche du talking blues, le fait de parler plutôt que de chanter lui permet de donner plus de poids à certains mots, comme il l'explique dans cet entretien :

Il y a quelque chose d'une liberté de rythme qui fait que tout d'un coup les mots prennent une liberté intérieure, prennent un poids, prennent une signification plus lourde, plus ample, plus à la hauteur de la dignité des mots, parce que, quand on parle, toi et moi, on crée l'emphase en faisant traîner sur une syllabe, en précipitant trois mots ici, en en ralentissant deux là. (Sarclo, 2018a)

Dans "Cauchemar Psychomoteur », on peut constater en effet que Sarclo s'affranchit du rythme régulier associé à la versification française, et cette façon d'interpréter la chanson lui permet de trâner davantage sur la fin du vers, avec une voix lubrique qui théâtralise la scène pour générer davantage de comique. Le narrateur refuse l'invitation de Rita avec les mots suivants: "Oh, no! no! / I've been through this movie before ", une métaphore qui signifie qu'il n'est pas né de la dernière pluie et n'a pas l'intention de se laisser duper, mais qui peut également être interprétée au pied de la lettre, à savoir qu'il a vu le film d'Alfred Hitchcock lorsqu'il est sorti quatre ans auparavant et qu'il sait très bien comment tout cela va se terminer pour lui. Aufray chante "Ce coup-là on me l'a déjà fait ", ce qui ne permet de transférer que le sens métaphorique. On pourrait imaginer une traduction du type « ce film-là, on me l'a déjà joué ", qui permettrait de conserver le double sens.

Dans le septième couplet, Rita parle de sa mère, "Rita mumbled something / 'Bout her mother on the hill» ["Rita marmonna quelque chose / Au sujet de sa mère sur la colline »], poursuivant ainsi le parallèle avec le personnage de Norman Bates. Aufray chante "Rita parla de sa mère / Qui dormait au cimetière ", ce qui n'est pas fidèle à la référence à Psychose, puisque justement la mère de Norman Bates n'a pas été enterrée, 
et son corps repose dans la maison sur la colline. De même, lorsque le narrateur dit, dans le dernier couplet, "Rita moved away / And got a job at a motel " ["Rita a déménagé / Et a trouvé un travail dans un motel »], Aufray chante «j'ai trouvé un boulot ", ce qui non seulement efface la référence au Bates Motel du film de Hitchcock, mais également change le sens du texte source de façon significative, puisque dans la version française c'est le narrateur qui a trouvé un travail. Sarclo a corrigé ce second aspect, chantant « elle a trouvé un boulot », mais la référence au motel, qui fait de cette chanson l'épisode précédent du film de Hitchcock, est perdue dans les deux cas. Enfin, dans le dernier vers, la référence au marécage citée plus haut, "Without freedom of speech / I might be in the swamp " ["sans la liberté de parole / Je serais peut-être dans le marais »], disparaît totalement dans la version chantée par Aufray, qui prend beaucoup de liberté dans la traduction des quatre derniers vers. Sarclo, en revanche, chante "Sans la liberté de parole / Je pourrirais dans l'étang », une traduction presque exacte de l'original. Le verbe " pourrir » est un étoffement intéressant, car il rend explicite l'évocation de l'image finale du film, où l'on voit la police extraire de l'eau stagnante la voiture contenant le cadavre de Marion Crane.

\section{L'Amérique conservatrice et la paranoïa anticommuniste}

Dans cette chanson, Dylan, avec un humour grinçant, établit un parallèle entre l'horreur du film d'Alfred Hitchcock et la terreur qui règne aux États-Unis à cause de la chasse aux sorcières contre les communistes occasionnée par la guerre froide. Avant de le faire de façon explicite à travers l'évocation du personnage de Fidel Castro, il installe l'atmosphère de paranoïa de façon plus subtile en dressant le portrait d'un vieux fermier conservateur, à qui le narrateur, alter ego du chanteur, sert de repoussoir. Lorsque le narrateur est accueilli avec un fusil, il se défend : «I'm a clean-cut kid / And I been to college too " [ "Je suis un garçon bien rangé / Et j'ai fait l'université »]. Aufray chante «En droit je suis licencié / J'ai fait l'université ». La référence aux études de droit est probablement une façon de transférer l'expression «clean-cut» du texte source en convoquant l'image d'un étudiant plutôt conservateur. Aufray et Delanoë utilisent ici habilement les représentations culturelles de l'auditeur français. On retrouve d'ailleurs cette image stéréotypée de l'étudiant en droit quelques années plus tard dans la chanson de Renaud, «Étudiant - poil aux dents » (1981) : «Étudiant en droit / Il y a plus de fachos dans ton bastion / Que dans un régiment de paras ». On notera que le mot «droit » peut également faire entendre l'expression « rester dans le droit chemin », qui est très proche du sens de « clean-cut » dans le texte de Dylan.

La façon dont le narrateur se défend pour persuader le fermier qu'il est honnête trouve son écho dans le couplet $4:$ "by the dirt 'neath my nails / I guess he knew I wouldn't lie " [«à en juger par la saleté sous mes ongles / Je suppose qu'il a compris que je ne lui mentirais pas »]. Ce trait d'humour cinglant de la part de Dylan est une manière de se moquer d'une certaine façon de penser attribuée au paysan, à savoir que quelqu'un qui travaille dur est nécessairement honnête. La traduction, « En voyant mes ongles sales / Il sut que je travaillais ", établit une relation entre les ongles sales et le travail, mais ne permet pas de rendre compte du lien de cause à effet supposé entre les deux qualités : travailleur, donc honnête. Les personnalités politiques des années 1960 avaient sans nul doute leurs propres expressions qui auraient pu en rendre compte, mais si l'on 
traduisait la chanson aujourd'hui, on pourrait imaginer utiliser une variation sur l'expression "la France qui se lève tôt ", utilisée par Nicolas Sarkozy dans son discours à Périgueux le 12 octobre 2006, et qui est probablement encore vive dans l'esprit de l'auditeur français. La rhétorique est en effet la même : opposer l'honnête travailleur au paresseux qui profite du système. L'objectif d'une telle traduction serait que l'auditeur français s'amuse d'entendre cette intrusion de la politique française dans le discours de Dylan, ce qui permettrait au moins de transférer l'effet comique. L'inconvénient de ce type de traduction est que la durée de vie de telles références à l'actualité est généralement très courte.

Dylan complète le portrait du fermier lorsque celui-ci jette un exemplaire du Reader's Digest à la tête du narrateur. Il s'agit d'un journal conservateur, ce qui est compréhensible pour la plupart des auditeurs américains des années 1960. On peut se demander, en revanche, si l'auditeur français perçoit cette subtilité, le journal étant beaucoup moins distribué en France. En plus de la ligne éditoriale du journal, le fait que celui-ci contienne des résumés d'œuvres célèbres est peut-être une façon pour Dylan de railler un fermier illettré qui n'est pas capable de lire des œuvres entières. On retrouve ce portrait d'une Amérique rurale conservatrice, sans instruction et facilement manipulable dans d'autres chansons de Dylan, notamment "Only a Pawn in Their Game » (1964b), qui traite de l'assassinat du militant noir Medgar Evers.

Le narrateur ayant promis de traire les vaches en échange de son hébergement, il ne peut pas quitter la ferme sans manquer à sa parole. Il préfère donc chercher un moyen de se faire jeter dehors et décide de crier "I like Fidel Castro and his beard " [ J'aime bien Fidel Castro et sa barbe »], comptant sur la réaction de son hôte, nécessairement anticommuniste, pour faire le reste. La formulation " and his beard" semble donner à cette affirmation un caractère ridicule, comme si l'on ne pouvait pas dire quoi que ce soit de positif au sujet de Fidel Castro en 1964, pas même sur sa barbe, sans être considéré comme un communiste. Cela ajoute sans nul doute au caractère comique de la chanson. On peut cependant considérer que, en plus de cet effet comique, Dylan faisait référence à l'apparence physique des hippies dans les années 1960, qui souvent portaient la barbe, comme Fidel Castro ou Che Guevara (Jenny, n. d.). Si l'on s'en tient à cette interprétation, cela revient à dire que le narrateur affirme être un membre radical de la contreculture. Aufray chante "J'ai crié "Fidel Castro / C'est un bon copain" ", produisant un effet comique à peu près équivalent, même si la barbe n'est pas mentionnée. D'une part, le narrateur affirme son éventuelle allégeance à l'idéologie de Castro, puisqu'il considère le personnage avec bienveillance, mais en plus le mot " copain", utilisé dans un contexte politique où on attendrait d'autres qualificatifs, crée un décalage lexical, ce qui peut provoquer le rire.

Les mots provocateurs du narrateur produisant l'effet escompté, la réaction du fermier est immédiate : " he came charging down the stairs / Sayin', "What's that I heard you say?" " [" il a descendu les escaliers quatre à quatre / En disant : “Qu'est-ce que je t'ai entendu dire là ?" »]. Le fermier insulte le narrateur : "You unpatriotic / Rotten doctor, Commie rat " [ « espèce de rat communiste / Docteur pourri, antipatriotique »]. Le mot «doctor » fait référence, non pas à l'exercice de la médecine, mais au titre universitaire que le narrateur prétend avoir obtenu lorsqu'il se présente au début de la chanson, ce qui explique la traduction «Espèce d'étudiant pourri » dans les deux versions françaises. Le fermier sort ensuite son fusil et chasse le narrateur, qui affirme " He wants to turn me in / To the F.B.I.» [«Il veut me dénoncer / au F.B.I. »]. Aufray chante « Espère bien me 
faire cueillir / Un jour par son F.B.I. ». L'utilisation du déterminant possessif «son » en français pour traduire l'article défini «the » devant «F.B.I. » est sans doute une façon pour Aufray et Delanoë de rendre explicite pour l'auditeur français la situation politique américaine, ce qui n'est pas nécessaire dans le texte source puisque cela fait partie du quotidien des auditeurs de Bob Dylan : si le narrateur est soupçonné d'être un agent communiste, il sera nécessairement poursuivi par le FBI, par conséquent l'organisme d'État est du côté du fermier, d'où le déterminant possessif.

Comme cela a déjà été mentionné plus haut, la traduction française de la conclusion par Aufray et Delanoë est pour le moins surprenante. Dylan chante: «Me, I romp and stomping / Thankful as I romp / Without freedom of speech / I might be in the swamp » [" Moi, je m'ébats et je cours joyeusement / Heureux de pouvoir le faire / Sans la liberté de parole / je serais peut-être dans le marais »]. Aufray chante un texte très différent : « Je continue à penser / Envers et contre tous / Sans liberté de parler / On est moins que rien du tout ». On retrouve, dans les deux premiers de ces quatre vers chantés par Aufray, l'idée de défiance contenue dans la chanson de Dylan, mais sans les qualités allitératives du texte d'origine, qui semblent faire entendre cette défiance. Les sonorités des mots " romp ", "stomping ", « romp » évoquent le trublion Dylan qui refuse de se soumettre à l'ordre établi. Concernant le dernier vers, l'absence de la référence à Psychose pose problème car celle-ci apporte une conclusion ironique à la narration. Le narrateur semble affirmer qu'il a été sauvé d'une mort certaine par la liberté de parole, alors qu'en réalité il a échappé de justesse aux coups de fusil du paysan qui voulait l'empêcher d'exprimer une opinion divergente. Sarclo traduit cette conclusion très différemment : «Et je me balade et je rigole / Bien heureux d'être content / Sans la liberté de parole / Je pourrirais dans l'étang ". La référence ironique à Psychose est intacte, et le caractère absurde du vers « Bien heureux d'être content » me semble assez bien correspondre, sinon à la lettre, du moins à l'esprit frondeur des mots « romp » and «stomp ».

\section{Le commis voyageur : un comique ancré dans l'espace et le temps}

Cette chanson, comme nous l'avons vu, présente une confrontation entre un "hillbilly» ["péquenaud / plouc / bouseux »] et un "city slacker» ["fainéant de la ville »], opposition qui fournit à Dylan une source d'humour. Ce type d'humour est déjà présent dans la chanson « Arkansas Traveler », un classique de la musique folk américaine, dont on trouve de nombreuses interprétations, et dans lequel le paysan se moque d'un citadin qui cherche son chemin (Seeger, 1958). Plus précisément, dans le cas de "Motorpsycho Nitemare », la confrontation entre ces deux personnages stéréotypés est empruntée aux blagues de commis voyageurs, qui étaient racontées aux États-Unis des années 1920 aux années 1960, et dont Dylan emprunte la structure pour la mélanger au scénario de Psychose (Attwood, 2015 : «Motorpsycho Nightmare: The Meaning of the Song and the Lyrics »). Cela est annoncé explicitement dès le deuxième couplet : «Are you that travelin' salesman / That I have heard about? » ["Êtes-vous ce commis voyageur / dont j'ai entendu parler?»]. Plus que de l'intertextualité, il s'agit de métatextualité, puisque le fermier, i.e. le personnage de la blague qui est toujours le dindon de la farce, annonce au narrateur dès le départ qu'il a déjà entendu ce type de blagues et qu'il est déjà sur ses gardes. 
La structure de ce type de blagues est toujours la même : un commis voyageur est perdu dans la campagne, comme le citadin de la chanson "Arkansas Traveler». Demandant asile pour la nuit à un fermier, il remarque que sa fille est extrêmement séduisante. Le fermier accepte de l'héberger mais pose toujours des conditions, notamment qu'il ne touche pas à sa fille. La chute de la blague repose toujours sur la façon dont le commis voyageur va réussir à déjouer la vigilance du fermier. L'un des traits distinctifs de ces blagues est l'exagération, une caractéristique empruntée aux blagues de cowboys. On retrouve d'ailleurs ces mêmes exagérations dans les talking blues de Woody Guthrie (1997, 1998a, 1998b), l'un des artistes qui a le plus influencé Bob Dylan. Guthrie vient lui-même d'une famille de cowboys, et a sans nul doute puisé dans ce type d'humour pour écrire ses talking blues (Klein, 1980:1). Un exemple d'exagération dans «Motorpsycho Nitemare » est la description de Rita, qui « semblait tout droit sortie de La Dolce Vita».

La traduction d'Aufray et Delanoë ne fait aucune référence au commis voyageur, qui est remplacé par un "espèce de vagabond / qui vient pour mendier ». Seule reste la méfiance du fermier à son égard, alors que le texte source est écrit comme une parodie d'une blague de commis voyageur, dans laquelle Rita, la fille du fermier, prend les devants et tente de séduire le narrateur, qui fait l'impossible pour lui échapper. Certaines des hyperboles qui caractérisent ce type d'humour, cependant, ont été conservées. Par exemple, lorsque le narrateur affirme «ten thousand miles / Today I drove » [« Aujourd'hui j'ai fait / 15000 kilomètres en voiture »], Hugues Aufray chante "j'ai fait huit cent bornes", ce qui constitue une hyperbole équivalente, puisque le "vagabond " de la version de Hugues Aufray est à pied, et non en voiture comme le serait un commis voyageur aux États-Unis. On notera que Sarclo a conservé le mot "vagabond» de la version d'Aufray, mais a dans un même temps été fidèle au texte source en ce qui concerne le moyen de locomotion du narrateur. En conséquence, lorsqu'il chante "j'ai fait huit cent bornes", cela ne constitue plus réellement une exagération et on perd un peu de l'humour du texte. A contrario, dans le premier couplet, lorsque le narrateur dit «I was mighty, mighty tired » [«j'étais complètement crevé »], Aufray chante "j'étais fatigué », ce qui est un euphémisme comparé au texte d'origine. Ici, c'est Sarclo qui rétablit l'exagération en chantant «J'étais carrément crevé ».

19 Cette modification effectuée par Sarclo soulève également une autre question concernant la version d'Aufray: l'infidélité au niveau de langue utilisé par Dylan. En effet, « mighty » est normalement un adjectif, qui signifie " puissant », « imposant », "énorme », dérivé du substantif «might» [ "puissance» / "force»] (Harrap's Unabridged Dictionary, 2007: 746). Par contre, il n'est utilisé comme adverbe pour modifier un adjectif que dans un registre familier, comme c'est le cas dans la chanson de Dylan. On pourra le traduire par "rudement", "vachement» ou "sacrément ", par exemple (ibid.). Par conséquent, l'expression «carrément crevé», utilisée par Sarclo, semble plus appropriée que « fatigué » pour reproduire le registre utilisé dans le texte source. Sarclo semble avoir prêté la plus grande attention au registre, puisqu'il utilise, dans ce même couplet, "J'ai cogné chez un fermier / Chez qui j'aurais pu pieuter ». On peut penser que le choix du registre familier n'est pas anodin, puisque la chanson est construite comme une blague de commis voyageur. Or, lorsqu'on raconte une blague, la proximité avec le public est parfois aussi importante que le contenu. Dylan a, dès le début de sa carrière, utilisé l'humour, que ce soit pendant ou entre les 
chansons, pour créer une complicité avec son public. Robert Shelton y fait référence dans No Direction Home: The Life and Music of Bob Dylan, parlant de « control of humor and timing " [ " contrôle de l'humour et du débit »] (2011 : chap. 3).

Deux autres exemples illustrent bien cette divergence de choix entre Aufray et Sarclo. Dans le cinquième couplet, où Dylan chante "I was sleeping like a rat " [ "je dormais comme un rat»], Aufray chante «je ne dormais que d'un œil». D'une part, il s'agit probablement d'un contresens, car si l'expression «sleep like a rat » n'existe pas en anglais, elle est proche d'une expression en français qui évoque un autre rongeur, "dormir comme un loir», que Sarclo a utilisée, et étant donné l'état de fatigue excessive dans lequel le narrateur prétend être au début de la chanson, il est probable que cette traduction soit plus proche de l'intention initiale de Dylan. D'autre part, Sarclo remplace le verbe " dormir " par " roupiller ", afin de poursuivre dans le même registre familier que le reste de son texte. Le second exemple de divergence entre les deux traductions concerne la traduction du mot «gun » dans le huitième couplet. Ce terme en anglais peut servir à désigner aussi bien une arme de poing qu'un canon, comme dans le titre du roman The Guns of Navarone (MacLean, 1957). Dans la chanson que nous étudions, il s'agit d'un fusil, comme l'indique le mot « rifle» dans le deuxième couplet. Aufray et Delanoë ont choisi le mot «escopette » alors que Sarclo a préféré " flingue ». Eu égard aux différentes considérations déjà évoquées, qui concernent le registre et le contenu humoristique de la chanson, les deux choix peuvent se défendre, et apportent une touche différente à la blague racontée par Dylan. D'un côté, avec "flingue ", on reste dans le registre familier. De l'autre, avec "escopette », Aufray utilise un mot que la plupart des auditeurs français ne comprendront probablement pas sans ouvrir un dictionnaire, mais qui, comme le mot "tromblon », évoque une arme ancienne, et par conséquent le côté rétrograde du fermier. Par ailleurs, "escopette " forme une paire minimale avec le mot «escampette». Le fermier prend son « escopette " tandis que le narrateur prend la poudre d'escampette. La sonorité de ce mot inhabituel nourrit l'humour de la chanson.

\section{Conclusion}

Comme nous avons pu le voir, l'une des difficultés, lorsqu'il s'agit de traduire une chanson de Bob Dylan, vient de l'intertextualité, qui est non seulement abondante, mais également très hétérogène. Dylan fait référence à des œuvres qui sont connues de la plupart de ses auditeurs américains, à l'actualité sociopolitique de son époque, et à des formes d'humour qui sont ancrées non seulement dans un territoire mais aussi dans une époque donnée. On peut bien sûr, comme l'ont fait Aufray et Delanoë, transformer le commis voyageur en vagabond et ne garder ainsi que la relation de méfiance entre le fermier et son visiteur. Il est possible d'obtenir ainsi un résultat qui conserve une bonne partie de l'humour de la version originale. Cependant, l'auditeur français a alors une perception déformée de l'œuvre de Dylan, car il pourrait avoir l'impression que cette histoire d'étudiant "vagabond » émane entièrement de l'imagination de Dylan, et ainsi passer totalement à côté de l'aspect parodique du texte source. La question qui se pose alors est la suivante : comment transférer cet aspect parodique en français pour un auditeur qui ne connaît pas l'œuvre parodiée? Une réponse pourrait être de parodier une œuvre sensiblement équivalente, connue du public français, mais il s'agit là d'une équation impossible, car aucune œuvre ne peut présenter tous les aspects 
parodiés par Dylan. Une autre solution est d'avoir recours à la parodie à plusieurs reprises dans le texte, en parodiant des œuvres différentes. C'est ce que j'ai proposé par exemple en citant un discours de Nicolas Sarkozy, pour montrer une convergence ponctuelle avec certains discours populistes présents aux États-Unis. En ce qui concerne les hyperboles, l'auditeur français n'ayant comme référence culturelle ni la blague de cowboys ni le talking blues de Woody Guthrie, on pourrait imaginer puiser dans un humour équivalent qui est la blague de Marseillais (cf. Gasquet-Cyrus, 2004 : 582). Enfin, pour ce qui est des références à Psychose et à La Dolce Vita, on peut raisonnablement penser qu'une bonne partie des auditeurs français possèdent ces références également. La chasse aux sorcières contre les communistes durant la guerre froide aux États-Unis est un phénomène qui n'a pas son équivalent en France, et c'est peut-être là l'obstacle majeur, étant donné que seule une situation politique nationale très tendue peut justifier la conclusion de la chanson, où le narrateur se fait violemment chasser de la ferme.

À ces difficultés, il faut ajouter l'aspect diachronique : la situation du traducteur n'est pas la même en 1965 et de nos jours. Certaines des références utilisées par Dylan peuvent être plus connues ou moins connues de l'auditeur français aujourd'hui. Ainsi, à l'obstacle que pose le décalage concernant les références lexico-culturelles entre un pays et l'autre s'ajoute la question de l'obsolescence d'une traduction. Sarclo donne une réponse très tranchée à ce sujet : «Je m'occupe des traductions 2018 , en sachant très bien leur durée de vie.» (Sarclo, 2018b) Enfin, même de façon synchronique, on peut s'interroger sur la notion d'« auditeur français ». Cela soulève une question qui dépasse le cadre de cet article : lorsque le traducteur produit un texte en français, il ne traduit pas nécessairement de la même façon pour le public d'Hugues Aufray qu'il le ferait pour le public de Cabrel, par exemple. Cet enjeu de réception peut expliquer en grande partie certaines différences dans les choix lexicaux entre Aufray et Sarclo dans "Cauchemar Psychomoteur». Il suffit d'entendre quelques extraits de l'œuvre personnelle de Sarclo pour constater qu'il écrit dans un registre beaucoup plus familier que celui qu'utilise habituellement Aufray. En outre, la langue d'Aufray est d'autant plus châtiée que sa version date de 1965. Une comparaison avec « Tout l'mond' un jour s'est planté » (Aufray, 1995), sa traduction de « Rainy Day Women \#12 \& 35 » (Dylan, 1966), permet de constater qu'il se permet, 30 ans plus tard, d'utiliser un registre beaucoup plus familier, ainsi que de nombreuses références à la drogue qui dépassent de loin le jeu sur le mot «stoned » de la version originale.

\section{BIBLIOGRAPHIE}

DANESI Marcel (2009), Dictionary of Media and Communications, Armonk, New York : M. E. Sharpe.

GASQUET-CYRUS Médéric (2004), Pratiques et représentations de l'humour verbal : étude sociolinguistique du cas marseillais (thèse de doctorat), Univ. Aix-Marseille 1, <http://www.academia.edu/1976689/ Pratiques_et_représentations_de_lhumour[...]>. 
GoscinNY René \& UdERZo Albert (1959), Astérix le Gaulois, Paris : Hachette.

Harrap's Unabridged Dictionary (2007), Édimbourg : Harrap.

KLEIN Joe (1980), Woody Guthrie: A Life, New York : Alfred A. Knopf, Distributed by Random House.

MACLEAN Alistair (1957), The Guns of Navarone, Londres : Collins.

MARGOTIN Philippe \& GUESDON Jean-Michel (2015), Bob Dylan: All the Songs. The Story behind Every Track (1 ${ }^{\text {re }}$ éd.), New York : Black Dog \& Leventhal Publishers.

RolLASON Christopher (2007), “"Sólo Soy un Guitarrista”: Bob Dylan in the Spanish-Speaking World--Influences, Parallels, Reception, and Translation », Oral Tradition, 22(1), 112-133, <https:// doi.org/10.1353/ort.2007.0012>.

SHELTON Robert (2011), No Direction Home: The Life and Music of Bob Dylan (ePub), Londres : Omnibus Press.

\section{Discographie}

Aufray Hugues (1965), « Cauchemar Psychomoteur », Aufray chante Dylan (LP), Barclay.

AUfRAY Hugues (1995), « Tout l'mond' un jour s'est planté », Aufray trans Dylan (CD), Arcade.

DyLAN Bob (1963), « Talkin' World War III Blues », The Freewheelin' Bob Dylan (LP), CBS.

DYLAN Bob (1964a), « Motorpsycho Nitemare », Another Side of Bob Dylan (LP), CBS.

DYLAn Bob (1964b), « Only a Pawn in Their Game », The Times They Are A-Changing (LP), Columbia.

DYLAN Bob (1966), « Rainy Day Women \#12 \& 35 », Blonde on Blonde (LP), CBS.

DYLAN Bob (2010), « Talkin' John Birch Paranoid Blues », The Bootleg Series, Vol. 9: The Witmark Demos 1962-1964 [Disc 1] (CD), Columbia.

GuTHRIE Woody (1997), « Talking Hard Work », This Land Is Your Land (The Asch Recordings, Vol. 1) (CD), Smithsonian Folkways.

GuTHRIE Woody (1998a), « Mean Talking Blues », Hard Travelin' (The Asch Recordings, Vol. 3) (CD), Smithsonian Folkways.

GuTHRIE Woody (1998b), « Talking Columbia », Hard Travelin' (The Asch Recordings, Vol. 3) (CD), Smithsonian Folkways.

RENAUD (1981), « Étudiant - poil aux dents », Le retour de Gérard Lambert (LP), Polydor.

SEEGER Pete (1958), « Arkansas Traveler », Pete Seeger and Sonny Terr (LP), Folkways Records.

\section{Filmographie}

CAPRA Frank (1944), Arsenic and Old Lace, Warner Bros.

Fellini Federico (1960), La Dolce Vita.

Hiтснсоск Alfred (1960), Psycho. Horror, Mystery, Thriller.

KUBRICK Stanley (1980), The Shining, Warner Bros.

SPIELBERG Steven (1971), Duel, Universal Pictures.

\section{Webographie}


ATTWOoD Tony (2015), « Motorpsycho Nightmare: The Meaning of the Song and the Lyrics ", en ligne sur Untold Dylan : <https://bob-dylan.org.uk/archives/1805> (consulté le 28 août 2018).

JeNNY (n. d.), « A Historical Look at Presidential Beards (and Facial Hair) », en ligne sur BC :

$<$ http://brocouncil.com/Bro-Council-Stuff/beard-of-the-month[...]>.

SARClo (2017), Cauchemar Psychomoteur, La Grange du Locle. <www.youtube.com/watch?

$\mathrm{v}=\mathrm{E} 1 \mathrm{~N} 85 \mathrm{lYy} 72 \mathrm{I}>$.

\section{Autres sources}

SARCLO (2018a), Interview à Lyon.

SARClo (2018b), « Durée de vie d'une traduction », 12 mars 2018.

STEVEnson Angus (2010), « Talking Blues », Oxford Dictionary of English ( $3^{\mathrm{e}}$ éd.), Oxford University

Press, Mac Dictionary, Version 2.2.2 (203).

\section{NOTES}

1. Toutes les traductions qui figurent entre crochets sont mes traductions.

2. En anglais, le son /əテ/ est considéré comme une diphtongue, i.e. un son unique.

\section{RÉSUMÉS}

La chanson "Motorpsycho Nitemare ", de Bob Dylan, a été enregistrée en français par Hugues Aufray dès 1965, un an après la sortie de l'original en langue anglaise. En 2018, Sarclo, un autre artiste francophone, en a enregistré une nouvelle version, utilisant la traduction d'Aufray et Delanoë, n'y apportant que quelques changements, et une interprétation bien différente. Cet article traite des obstacles que comporte la traduction d'une chanson qui regorge de références à la culture états-unienne, parmi lesquelles certaines dont l'auditeur français a peu de chance d'avoir connaissance. La question de l'interprétation est également capitale, car celle-ci influe sur la perception de l'humour de la chanson.

The song « Motorpsycho Nitemare ", by Bob Dylan, was recorded in French by Hugues Aufray as early as 1965, one year after its original release in English. In 2018, Sarclo, another Frenchspeaking performer, recorded a new version, using Aufray and Delanoë's translation, with only a few changes, yet a very different performance. This article deals with the obstacles involved in the translation of a song which is full of references to American culture, some of which the French listener is unlikely to be familiar with. The issue of performance is important too, as it bears on the way the humour of the song is received. 
INDEX

Mots-clés : Bob Dylan, traduction, chanson, Psychose

Keywords : Bob Dylan, translation, song, Psycho

AUTEUR

JEAN-CHARLES MEUNIER

Université polytechnique des Hauts-de-France 